\title{
Malnutrition screening and acute kidney injury in hospitalised patients: a retrospective study over a 5-year period from China
}

\author{
Chenyu $\mathrm{Li}^{1}$, Lingyu Xu ${ }^{1}$, Chen Guan ${ }^{1}$, Long Zhao ${ }^{1}$, Congjuan Luo ${ }^{1}$, Bin Zhou ${ }^{1}$, Xiaosu Zhang ${ }^{1}$, Jing Wang ${ }^{2}$, \\ Jun Zhao ${ }^{3}$, Junyan Huang ${ }^{4}$, Dan $\mathrm{Li}^{4}$, Hong Luan ${ }^{1}$, Xiaofei Man ${ }^{1}$, Lin Che ${ }^{1}$, Yanfei Wang ${ }^{1}$, Hui Zhang ${ }^{1}$ and \\ Yan $\mathrm{Xu}^{1 *}$ \\ ${ }^{1}$ Department of Nephrology, Affiliated Hospital of Qingdao University, Qingdao 266003, People's Republic of China \\ ${ }^{2}$ Renal Department of Internal Medicine, Haiyang People's Hospital, Haiyang 265100, People's Republic of China \\ ${ }^{3}$ Department of Nephrology, Shandong Weifang People's Hospital, Weifang 261000, People's Republic of China \\ ${ }^{4}$ Department of Nephrology, Qingdao Central Hospital, Qingdao 266003, People's Republic of China
}

(Submitted 19 February 2019 - Final revision received 7 October 2019 - Accepted 18 October 2019)

\begin{abstract}
Malnutrition and acute kidney injury (AKI) are common complications in hospitalised patients, and both increase mortality; however, the relationship between them is unknown. This is a retrospective propensity score matching study enrolling 46549 inpatients, aimed to investigate the association between Nutritional Risk Screening 2002 (NRS-2002) and AKI and to assess the ability of NRS-2002 and AKI in predicting prognosis. In total, 37190 (80 \%) and 9359 (20\%) patients had NRS-2002 scores $<3$ and $\geq 3$, respectively. Patients with NRS-2002 scores $\geq 3$ had longer lengths of stay $(12.6(\mathrm{sD} 7.8) v .10 \cdot 4(\mathrm{sD} 6.2) \mathrm{d}, P<0.05)$, higher mortality rates $(9.6 v .2 .5 \%, P<0.05)$ and higher incidence of AKI $(28 v .16 \%$, $P<0.05)$ than patients with normal nutritional status. The NRS-2002 showed a strong association with AKI, that is, the risk of AKI changed in parallel with the score of the NRS-2002. In short- and long-term survival, patients with a lower NRS-2002 score or who did not have AKI achieved a significantly lower risk of mortality than those with a high NRS-2002 score or AKI. Univariate Cox regression analyses indicated that both the NRS-2002 and AKI were strongly related to long-term survival (AUC 0.79 and 0.71 ) and that the combination of the two showed better accuracy (AUC 0.80 ) than the individual variables. In conclusion, malnutrition can increase the risk of AKI and both AKI and malnutrition can worsen the prognosis that the undernourished patients who develop AKI yield far worse prognosis than patients with normal nutritional status.
\end{abstract}

Key words: Nutritional Risk Screening 2002: Acute kidney injury: Propensity score matching

Acute kidney injury (AKI) is a severe hospital-acquired condition caused by a range of factors in various clinical settings and is associated with kidney function deterioration and increased mortality ${ }^{(1)}$. The worldwide annual incidence of AKI is approximately $2100 /$ million worldwide ${ }^{(2)}$, and the number of AKIcaused deaths is approximately 2 million $^{(3)}$, of which $20-25 \%$ are inpatients; in particular, critical AKI has a mortality rate of more than $50 \%$ during dialysis treatment ${ }^{(4)}$. Despite advances in renal replacement therapy technologies, patients with AKI still face a high risk of sequelae, including irreversible acute damage with the possibility of progression to chronic kidney disease (CKD) and other complications ${ }^{(5,6)}$. In view of the lack of effective predictive factors, active treatment and unpredictable prognoses associated with AKI, prevention and risk factor management remain critical.
Malnutrition is defined as any nutritional imbalance, which is a major complication in hospitalised patients and increases the length of stay (LOS), healthcare costs and mortality ${ }^{(7-11)}$. The prevalence of malnutrition is $15-80 \%$ in hospitalised patients, while only $3 \%$ could be diagnosed and treated ${ }^{(8,12)}$. It has been reported that over $40 \%$ of CKD patients are undernourished ${ }^{(13)}$ and uraemia and dialysis can impact the nutritional status of patients with kidney disease ${ }^{(14,15)}$. AKI as the spread of inflammatory processes from the kidney to other organ systems is also affected by metabolic changes and undernutrition. Therefore, undernourished patients are more vulnerable to $\mathrm{AKI}^{(16)}$ and many factors such as hypoproteinaemia and insulin resistance are involved in this progression ${ }^{(17)}$. Good nutritional support has been advocated in AKI, whether treated with or without RRT. In animal experiments, enteral $v$. parenteral nutrition has

Abbreviations: AKI, acute kidney injury; CKD, chronic kidney disease; LOS, length of stay; NRS-2002, Nutritional Risk Screening 2002; PSM, propensity score matching; Scr, serum creatinine.

* Corresponding author: Yan Xu, email xuyanqyfy@126.com 


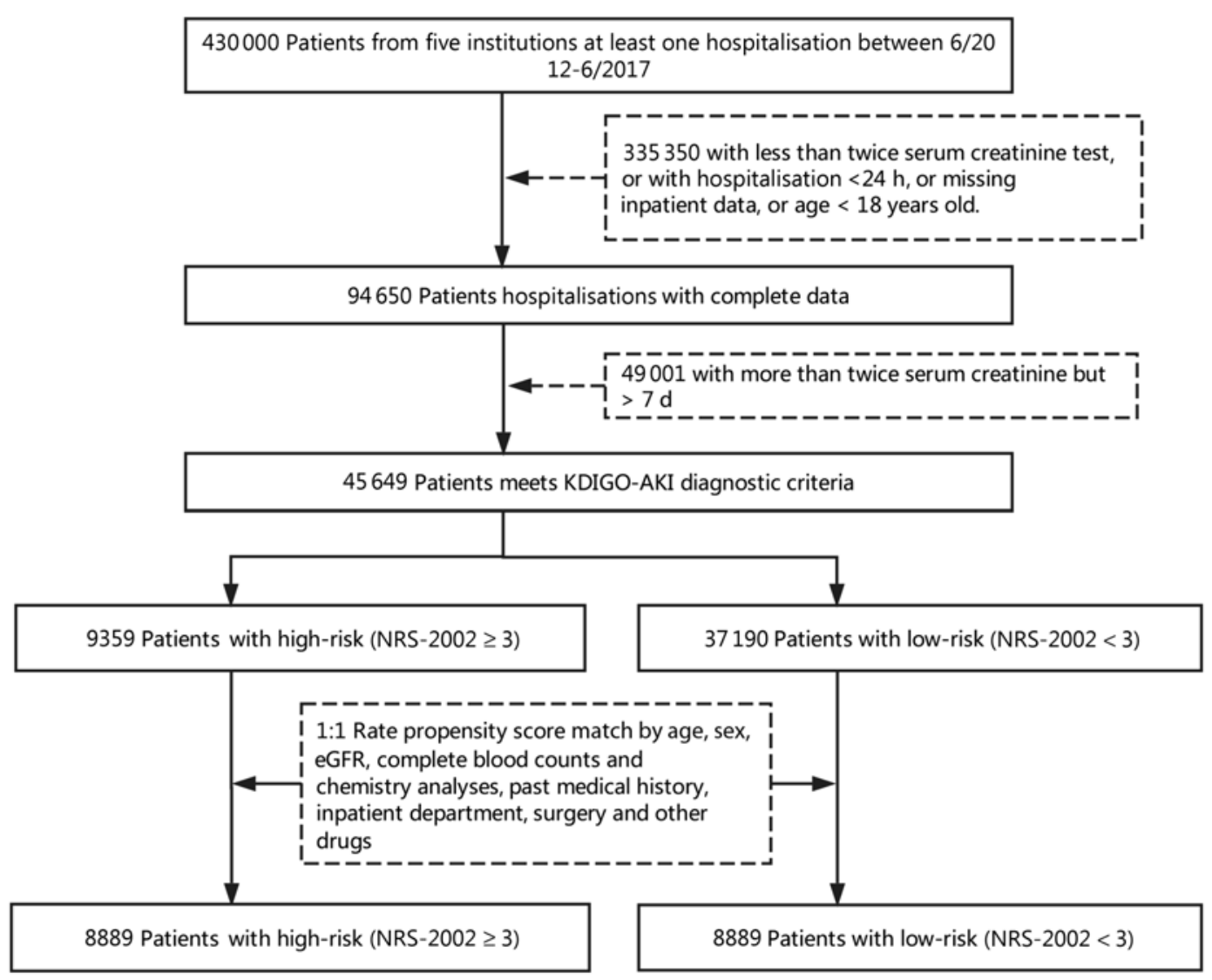

Fig. 1. Flow diagram of patient selection. KDIGO, Kidney Disease Improving Global Outcomes; AKI, acute kidney injury; NRS-2002, Nutritional Risk Screening 2002; eGFR, estimated glomerular filtration rate.

been shown to alleviate $\mathrm{AKI}^{(18)}$ and increased protein intake reduces tubular injury ${ }^{(19,20)}$. In patients with acute renal failure, protein and amino acids augment renal perfusion and improve renal function ${ }^{(21)}$. A randomised controlled trial studying the effects of daily intravenous amino acid supplementation in critically ill patients showed a significant increase in estimated glomerular filtration rate in the treatment group $^{(22)}$. However, the nutrition status is often neglected in AKI patients; therefore, accurate determination of nutritional status and providing nutritional support remain a challenging task in the treatment of $\mathrm{AKI}^{(23,24)}$.

The Nutritional Risk Screening 2002 (NRS-2002), developed by the European Society for Clinical Nutrition and Metabolism $(\text { ESPEN })^{(25)}$, has been widely used in clinical malnutrition screening and validated for assessing the nutritional status of patients for nutritional intervention in a variety of diseases ${ }^{(26,27)}$ including CKD $^{(13,28)}$. The NRS-2002 was composed of a score for impaired nutritional status, a score for the severity of disease and an additional score if age $\geq 70$ years ${ }^{(25)}$, which recognise malnutrition if total score of NRS-2002 $\geq 3^{(29)}$. Those NRS-2002 components such as low BMI, major abdominal operation and intensive care admission are also recognised risk factors for the occurrence of AKI; however, the relationship between the entirety NRS-2002 and AKI is unknown. Therefore, the aim of the present study was to (1) study the amounts of malnutrition in AKI patients, (2) investigate the relationship between NRS-2002 and AKI and (3) assess the ability of NRS-2002 and AKI to predict prognosis in hospitalised patients, all of which will contribute to the prediction, prevention and treatment of AKI.

\section{Materials and methods \\ Study design and procedure}

This is a retrospective propensity score matching (PSM) study which includes all hospital admissions except obstetrics departments. A total of 430000 inpatients from five institutions admitted between 1 June 2012 and 31 December 2017 were enrolled in the present study. Fig. 1 describes the selection of patients in the final analysis, and 46549 consecutive inpatients were eligible. Patients were excluded if they met one of the following characteristics:

(1) Patients with age $<18$ years old.

(2) Patients with hospitalisation $<24 \mathrm{~h}$.

(3) Patients with less than twice serum creatinine (Scr) test.

(4) Patients requiring dialysis before or after hospitalisation.

(5) Patients with missing data elements which needed further analysis.

The present study was approved by the Institutional Review Board (IRB, QDFY WZ 2018-9-13) to screen out those patients with qualifying Scr levels. All subjects were treated with standard care without intervention from the present study and followed 
up for 5-63 months until 1 January 2019, with a median time of 14 months. Clinical data were obtained via electronic medical records and a database review and were de-identified (patient names were replaced with the identification code, and private patient information was deleted before analysis) to protect patient privacy. IRB supervised the entire de-identified process; thus, each patient's informed consent is waived.

All patients underwent malnutrition risk screening by using the NRS-2002 within the first $24 \mathrm{~h}$ after admission. Complete blood counts, blood chemistry analyses and urine tests were performed within $3 \mathrm{~d}$ of admission. In addition, necessary information including demographic characteristics (sex, age and BMI), inpatient department (medical, surgical, oncology, gynaecology or intensive care unit) and hospitalisation-related factors (LOS, surgery and number of deaths) was collected. Patients' co-morbidities (hypertension, diabetes mellitus, CHD and CKD) were all defined according to International Classification of Diseases (ICD) 10th Revision (https://apps. who.int/iris/handle/10665/42980) in our hospital. The information was extracted from an electronic clinical letter via regular expression matching.

\section{Diagnostic criteria}

The diagnostic classification and criteria of AKI were based on Kidney Disease: Improving Global Outcomes 2012 as follows: (1) Scr level $>26.5 \mathrm{mmol} / 1$ within $48 \mathrm{~h}$; (2) an increase in Scr to more than 1.5 -fold the baseline-confirmed value or an increase presumed to have occurred within $7 \mathrm{~d}$ or (3) urine output $<0.5 \mathrm{ml} / \mathrm{kg}$ per $\mathrm{h}$ for more than $6 \mathrm{~h}$. AKI staging was defined according to the Kidney Disease: Improving Global Outcomes guidelines. Baseline Scr was defined as the first Scr value measured during hospitalisation. The AKI diagnosis time was established once the participants first met the Kidney Disease: Improving Global Outcomes criteria. The baselineestimated glomerular filtration rate was calculated using the CKD-Epidemiology Collaboration formula ${ }^{(30)}$.

Nutritional status during hospitalisation was evaluated by the NRS-2002 scale, which consists of a score for impaired nutritional status, a score for the severity of disease and an additional score if age $\geq 70$ years. Additionally, impaired nutritional status is scored from 0 to 3 according to changes in BMI, weight and food intake. The severity of disease is scored from 0 to 3 based on different kinds of diseases according to the individual medical history and age records. Moreover, an additional 1 point should be added to the total score for patients aged over 70 years. A total score of $\geq 3$ indicated nutritional impairment. The NRS-2002 was validated in the Chinese population ${ }^{(31,32)}$.

\section{Statistical analysis}

We choose an empirical threshold that indicators with more than $15 \%$ missing values were excluded from data. In addition, indicators with less than $15 \%$ missing values warranted interpolation by multiple imputation using the mice package ${ }^{(33)}$, and all model variables were considered simultaneously. We assumed that the data were missing at random ${ }^{(34)}$; therefore, we performed predictive mean matching ${ }^{(35)}$ to generate five complete imputed data sets that fit the logistic models. Sample size calculation showed that an estimated 442 AKI-free events would be needed to provide $90 \%$ power for detecting a minimum clinically meaningful OR of 0.64 for NRS-2002 scores $<3$ relative to NRS-2002 scores $\geq 3$ with a two-sided $\alpha$ of $0 \cdot 05$. Continuous variables were transformed into categorical variables based on recognised cutoff values.

To account for measurement confounding, we performed 1:1 rate PSM based on selected covariates using logistic regression with a caliper of 0.2 of the standard deviation ${ }^{(36)}$. The PSM was performed for the NRS-2002 scores $<3$ and $\geq 3$ matched patients and stratified by the baseline characteristics and other variables related to hospitalisation, such as the inpatient department, surgery and concomitant drugs. Forward stepwise regression analyses were performed to select adjusted indicators for logistics and Cox regression models. The Kaplan-Meier method was used to study the effects of NRS-2002 and AKI on short- and long-term prognosis among the inpatients, whereas the Mantel-Haenszel log-rank test was performed to evaluate differences among the groups classified by the NRS-2002 and AKI stage. The results were compared using the $\chi^{2}$ test or Fisher's exact test. Continuous variables were compared using a $t$ test or the Mann-Whitney $U$ test for variables with a nonnormal distribution. The AUC of the receiver operating characteristics was compared by using the DeLong method. All analyses were performed using $\mathrm{R}$ version 3.4 .2 (http:// www.r-project.org/).

\section{Results}

\section{Patient characteristics and propensity score matching}

In total, 46549 consecutive inpatients were eligible for final analysis, in which 7175 (15\%) patients had AKI and 39374 (85\%) patients were AKI-free. Before PSM, 37190 (80\%) and 9359 (20\%) patients had NRS-2002 scores $<3$ and $\geq 3$, respectively. The patients with NRS-2002 scores $\geq 3$ had higher concentrations of leucocytes, aspartate aminotransferase and alanine aminotransferase but lower $\mathrm{Hb}$ and estimated glomerular filtration rate and had increased use of antibiotics and surgical operation than those in the NRS-2002 scores <3 group (Table 1). Additionally, the incidence of AKI was $31 \%$ in the NRS-2002 scores $\geq 3$ group, which was higher than $12 \%$ in the NRS-2002 scores $<3$ group. The NRS-2002 scores (3.2 (sD 1.6) v. $2 \cdot 5$ (sD 1.1), $P<0 \cdot 05$ ) in AKI group as well as the components nutrition score and disease severity score are higher than those without AKI (online Supplementary Table S1). Therefore, patients with scores $\geq 3$ or AKI seemed to be more likely to suffer severe disease and worse liver or kidney function.

To eliminate this bias, we used PSM to match two more comparable 1:1 rate cohorts. After PSM, NRS-2002 scores $<3$ and $\geq 3$ groups were well balanced on most characteristics, except the incidence of AKI which was still higher in the NRS-2002 scores $\geq 3$ group than in the $<3$ group (29v. $16 \%, P<0.05$, Table 1 ). Moreover, we compared the short-term prognosis between NRS-2002 scores $<3$ group and $\geq 3$ group after PSM. The results showed patients with NRS-2002 scores $\geq 3$ had longer LOS (12.6 (sD 7.8) v. 10.4 (sD 6.2) d, $P<0.05$ ), higher mortality rates (9.6 v. $2.5 \%, P<0.05)$ and higher rates of AKI than the patients 
Table 1. Characteristics of inpatients before and after propensity score matching (Mean values and standard deviations; numbers of patients and percentages)

\begin{tabular}{|c|c|c|c|c|c|c|c|c|c|c|c|c|c|}
\hline \multirow[b]{4}{*}{ Variables } & \multicolumn{13}{|c|}{ Propensity score weighting ${ }^{*}$} \\
\hline & \multicolumn{6}{|c|}{ Before } & \multicolumn{7}{|c|}{ After } \\
\hline & \multicolumn{2}{|c|}{$\begin{array}{c}\text { NRS-2002<3 } \\
\quad(n 37190)\end{array}$} & \multicolumn{3}{|c|}{$\begin{array}{l}\text { NRS-2002 } \geq 3 \\
\quad(n \text { 9359) }\end{array}$} & \multirow[b]{2}{*}{$P$} & \multicolumn{3}{|c|}{$\begin{array}{l}\text { NRS-2002<3 } \\
\quad(n \text { 8889) }\end{array}$} & \multicolumn{3}{|c|}{$\begin{array}{l}\text { NRS }-2002 \geq 3 \\
\quad(n \text { 8889) }\end{array}$} & \multirow[b]{2}{*}{$P$} \\
\hline & Mean & SD & Mean & & SD & & Mean & & $\mathrm{SD}$ & Mean & & SD & \\
\hline \multirow{2}{*}{\multicolumn{14}{|c|}{ Baseline characteristics }} \\
\hline & \multirow{3}{*}{\multicolumn{2}{|c|}{$\begin{array}{c}20609 \\
55\end{array}$}} & & & & & & & & & & & \\
\hline$n$ & & & \multirow{2}{*}{\multicolumn{3}{|c|}{$\begin{array}{c}5765 \\
62\end{array}$}} & $<0.05$ & \multirow{2}{*}{\multicolumn{3}{|c|}{$\begin{array}{c}5333 \\
60\end{array}$}} & & 5386 & & 0.16 \\
\hline$\%$ & & & & & & & & & & & 60 & & \\
\hline Age (years) & 56 & 14 & 64 & & 16 & $<0.05$ & 57 & & 16 & 63 & & 16 & $<0.05$ \\
\hline $\mathrm{BMI}\left(\mathrm{kg} / \mathrm{m}^{2}\right)$ & 25 & 3 & 22 & & 4 & $<0.05$ & 25 & & 4 & 23 & & 4 & $<0.05$ \\
\hline ALB $(g / l)$ & 38 & 6 & 34 & & 7 & $<0.05$ & 36 & & 7 & 34 & & 7 & $<0.05$ \\
\hline Leucocytes $\left(\times 10^{9} /\right.$ litre $)$ & 6.9 & 3.0 & $8 \cdot 1$ & & $4 \cdot 3$ & $<0.05$ & 7.7 & & 3.7 & 7.9 & & $4 \cdot 1$ & 0.30 \\
\hline $\mathrm{Hb}(\mathrm{g} / \mathrm{l})$ & 130 & 21 & 118 & & 25 & $<0.05$ & 120 & & 24 & 119 & & 24 & 0.76 \\
\hline PLT $\left(\times 10^{9} /\right.$ litre $)$ & 221 & 77 & 222 & & 97 & $<0.05$ & 220 & & 88 & 224 & & 96 & 0.75 \\
\hline ALT (U/I) & 35 & 84 & 49 & & 133 & $<0.05$ & 43 & & 114 & 47 & & 125 & 0.05 \\
\hline AST (U/I) & 30 & 79 & 55 & 184 & & $<0.05$ & 39 & & 110 & 51 & & 170 & 0.13 \\
\hline UA $(\mu \mathrm{mol} / /)$ & 309 & 109 & 284 & & 135 & $<0.05$ & 299 & & 117 & 283 & & 132 & 0.21 \\
\hline eGFR (ml/min) & 105 & 29 & 96 & & 34 & $<0.05$ & 102 & & 36 & 98 & & 32 & 0.62 \\
\hline Clinical intervention & & & & & & & & & & & & & \\
\hline ACEI & & & & & & & & & & & & & 0.54 \\
\hline$n$ & & & & 572 & & $<0.05$ & & 541 & & & 544 & & \\
\hline$\%$ & & & & $6 \cdot 0$ & & & & $6 \cdot 1$ & & & $6 \cdot 1$ & & \\
\hline ARB & & & & & & & & & & & & & 0.37 \\
\hline$n$ & & & & 1089 & & 0.07 & & 1050 & & & 1052 & & \\
\hline$\%$ & & & & 12 & & & & 12 & & & 12 & & \\
\hline CCB & & & & & & & & & & & & & 0.45 \\
\hline$n$ & & & & 2437 & & 5005 & & 2258 & & & 2263 & & \\
\hline$\%$ & & & & 26 & & $<0.05$ & & 25 & & & 25 & & \\
\hline NSAID & & & & & & $<0.05$ & & & & & & & 0.54 \\
\hline$n$ & & & & 2011 & & & & 1968 & & & 1983 & & \\
\hline$\%$ & & & & 21 & & & & 22 & & & 22 & & \\
\hline Antibiotic & & & & & & & & & & & & & 0.06 \\
\hline$n$ & & & & 6990 & & $<0.05$ & & 6451 & & & 6544 & & \\
\hline$\%$ & & & & 75 & & & & 72 & & & 74 & & \\
\hline Surgical & & & & & & & & & & & & & 0.59 \\
\hline$n$ & & & & 5335 & & $<0.05$ & & 5333 & & & 5244 & & \\
\hline$\%$ & & & & 57 & & & & 60 & & & 59 & & \\
\hline ICU & & & & & & & & & & & & & \\
\hline$n$ & & & & 1466 & & _- & & 0 & & & 1066 & & _- \\
\hline$\%$ & & & & 16 & & & & 0 & & & 12 & & \\
\hline Co-morbidities & & & & & & & & & & & & & \\
\hline CKD & & & & & & & & & & & & & \\
\hline$n$ & & & & 666 & & $<0.05$ & & 506 & & & 518 & & 0.97 \\
\hline$\%$ & & & & $7 \cdot 1$ & & & & $5 \cdot 7$ & & & $5 \cdot 8$ & & \\
\hline DM & & & & & & & & & & & & & \\
\hline$n$ & & & & 1869 & & $<0.05$ & & 1717 & & & 1702 & & 0.06 \\
\hline$\%$ & & & & 20 & & & & 19 & & & 19 & & \\
\hline $\mathrm{HBp}$ & & & & & & & & & & & & & \\
\hline$n$ & & & & 3211 & & $<0.05$ & & 2979 & & & 2997 & & 0.47 \\
\hline$\%$ & & & & 34 & & & & 34 & & & 33 & & \\
\hline $\mathrm{CHD}$ & & & & & & & & & & & & & \\
\hline$n$ & & & & 1841 & & $<0.05$ & & 1639 & & & 1673 & & 0.51 \\
\hline$\%$ & & & & 20 & & & & 18 & & & 19 & & \\
\hline LOS and $\mathrm{AKI}$ & & & & & & & & & & & & & \\
\hline $\begin{array}{l}\text { LOS (d) } \\
\text { AKI }\end{array}$ & 9.9 & $5 \cdot 6$ & $12 \cdot 6$ & & $8 \cdot 0$ & $<0.05$ & $10 \cdot 4$ & & $6 \cdot 2$ & $12 \cdot 6$ & & $7 \cdot 8$ & $<0.05$ \\
\hline$n$ & & & & 2856 & & $<0.05$ & & 1425 & & & 2556 & & $<0.05$ \\
\hline$\%$ & & & & 31 & & & & 16 & & & 29 & & \\
\hline
\end{tabular}

NRS-2002, Nutritional Risk Screening 2002; ALB, albumin; PLT, platelet; ALT, alanine transaminase; AST, aspartate aminotransferase; UA, uric acid; eGFR, estimated glomerular filtration rate; ACEI, angiotensin-converting-enzyme inhibitors; ARB, angiotensin II receptor blockers; CCB, Ca channel blockers; NSAID, non-steroidal anti-inflammatory drugs; ICU, intensive care unit; CKD, chronic kidney disease; DM, diabetes mellitus; HBp,

hypertension; LOS, length of stay; AKI, acute kidney injury.

*Age, BMI, ALB and AKI were not used for covariate balance. 
Table 2. Nutritional Risk Screening 2002 (NRS-2002) and acute kidney injury (AKI) in first $7 \mathrm{~d}$ and whole hospitalisation after propensity score matching

(Numbers of patients and percentages; mean values and standard deviations)

\begin{tabular}{|c|c|c|c|c|c|}
\hline & \multicolumn{2}{|c|}{$\begin{array}{l}\text { NRS-2002 < } 3 \\
\quad(n \text { 8889) }\end{array}$} & \multicolumn{2}{|c|}{$\begin{array}{l}\text { NRS }-2002 \geq 3 \\
\quad(n \text { 8889) }\end{array}$} & \multirow[b]{2}{*}{$P$} \\
\hline & $n$ & $\%$ & $n$ & $\%$ & \\
\hline $\begin{array}{l}\text { AKI during } \\
\text { hospital stay }\end{array}$ & 1425 & 16 & 2556 & 29 & $<0.05$ \\
\hline Stage 1 & 1298 & 15 & 2269 & 25 & \\
\hline Stage 2 & 100 & $1 \cdot 1$ & 201 & $2 \cdot 3$ & \\
\hline Stage 3 & 27 & 0.3 & 86 & 1.0 & \\
\hline$A K I$ in first $7 d$ & 1071 & 12 & 1665 & 18 & $<0.05$ \\
\hline Stage 1 & 995 & 11 & 1500 & 16 & \\
\hline Stage 2 & 57 & 0.6 & 110 & 1.2 & \\
\hline Stage 3 & 19 & 0.2 & 55 & 0.6 & \\
\hline Dead in $60 \mathrm{~d}$ & 218 & $2 \cdot 5$ & 853 & 9.6 & $<0.05$ \\
\hline \multicolumn{6}{|l|}{$\operatorname{LOS}(\mathrm{d})$} \\
\hline Mean & & $10 \cdot 4$ & & $12 \cdot 6$ & $<0.05$ \\
\hline SD & & $6 \cdot 2$ & & $7 \cdot 8$ & \\
\hline
\end{tabular}

LOS, length of stay.

with normal nutritional status (Table 2). Since NRS-2002 requires weekly assessment, we investigated the condition within $7 \mathrm{~d}$ after the NRS-2002 assessment and showed that the incidence of AKI in the NRS-2002 scores $\geq 3$ group was higher than that in the NRS-2002 $<3$ group $(18.73$ v. $2.05 \%, P<0.05$, Table 1$)$, suggesting that NRS-2002 could also be associated with AKI during early hospitalisation.

\section{Nutritional Risk Screening 2002 components are associated with acute kidney injury}

To better identify whether malnutrition increases the risk of AKI and study the relationship of NRS-2002 components and AKI, both univariate and multivariate logistic regression were performed (Table 3). As a result, the total score of NRS-2002 showed a strong association with AKI, that is, the risk of AKI changed in parallel with the NRS-2002 score $\left(P_{\text {for trend }}<0.05\right.$, 0.67 AUC for the univariate model, 0.78 for the multivariate model), suggesting that undernutrition represents a major negative factor for AKI. In the NRS-2002 component, albumin (ALB) was the most important nutritional variable associated with AKI (AUC 0.67). Other parts of the NRS-2002, such as the severity of disease (AUC 0.63), impaired nutritional status (AUC 0.59) and age (AUC 0.539), all slightly contributed to the risk of AKI but were not as significant as the total score of the NRS-2002 (Table 3). Moreover, we studied the relationship between NRS-2002 and AKI in different clinical situations. The results showed these associations are constant across different sex, surgical status and history of diabetes mellitus and hypertension, indicating a good generalisability association that undernutrition increases the risk of AKI (Fig. 2).

\section{Both acute kidney injury and malnutrition increased the risk of mortality}

We further study the effects of NRS-2002 and AKI on short- and long-term mortality. Patients with NRS-2002 scores $<3$ achieved significantly better long-term survival than those with higher scores $(P<0.05)$, suggesting that malnutrition was associated with adverse prognosis of inpatients (Fig. 3(a)). Similarly, AKI during hospitalisation significantly reduced survival, and survival worsened in patients with higher AKI stages (Fig. 3(b)). Therefore, both malnutrition identified by NRS-2002 and AKI were negative prognostic factors for short- and long-term survival. Moreover, the risk of mortality of non-AKI patients with NRS-2002 scores $\geq 3$ was higher than that of patients with NRS-2002 scores $<3$ (HR 6.47, 6.06-6.90, $P<0 \cdot 05$ ). Similar outcomes were noted in AKI patients with NRS-2002 scores $<3$ (HR $6 \cdot 74,6 \cdot 28-7 \cdot 24, P<0 \cdot 05)$, and AKI patients with NRS-2002 scores $\geq 3$ had the worst prognosis as expected (HR 24.8, 23.3-26.3, Fig. 3(c), Table 4), suggesting that NRS-2002 scores $\geq 3$ or AKI was associated with higher risk of mortality of patients. Furthermore, we studied the predictive ability of AKI and NRS-2002 for mortality by using both univariate and multivariate Cox regression analysis. Univariate Cox regression (Table 4) showed that the NRS-2002 was strongly associated with longterm survival (AUC $0.79, P<0.05$ ) and was better than AKI (AUC $0.71, P<0.05$ ). The combination of the NRS-2002 and AKI exhibited higher accuracy than the individual variables (AUC 0.80, $P<0.05$ ). However, multivariate Cox regression analysis revealed that the NRS-2002 scores were not the direct factor that increased mortality when the scores were $\geq 3$ (Table 4). Since the death events of patients mainly occurred during hospitalisation, the short-term prognosis of patients was similar to the long-term prognosis (Fig. 3(d)-(f) and Table 4).

\section{Discussion}

Malnutrition screening is a simple and efficient tool for identifying the risk of malnutrition and helping patients receive timely and effective intervention in the hospital ${ }^{(25,37)}$. Many nutrition screening tools are available, but European Society for Clinical Nutrition and Metabolism recommends the use of the NRS2002 in hospitalised patients. However, European Society for Clinical Nutrition and Metabolism also recommends a malnutrition universal screening tool for adults in community settings and a mini nutritional assessment short form for older adults. Due to the lack of a gold standard tool for assessing the risk of malnutrition, different approaches have been used in published studies. However, the NRS-2002 is more flexible, accurate and straightforward for use in clinical practice. To the best of our knowledge, our study is the first to evaluate the association between the NRS-2002 and AKI in hospitalised patients and to estimate the ability of NRS-2002 and AKI in predicting prognosis.

Malnutrition and AKI are common complications in hospitalised patients and increase LOS and mortality, but the nutritional status of patients as it relates to AKI is poorly described in the literature. In our study, 46549 inpatients from five institutions were enrolled between 1 June 2012 and 31 December 2017 and followed up for 5-63 months. The patients with NRS-2002 scores $\geq 3$ had a greater risk of AKI and longer LOS and higher mortality rates than the patients with normal nutritional status, which was consistent with previous studies that undernourished 
Table 3. Logistic regression model including variables associated with malnutrition as assessed by the Nutritional Risk Screening 2002 (NRS-2002) and corresponding risk for acute kidney injury (AKI) (Odds ratios and interquartile ranges (IQR))

\begin{tabular}{|c|c|c|c|c|c|c|c|c|}
\hline NRS-2002 & OR & IQR & $P$ & AUC & Adjusted OR* & IQR & $P$ & AUC \\
\hline \multicolumn{9}{|l|}{ Total score } \\
\hline 0 & 0.28 & $0.27,0.29$ & $<0.05$ & 0.67 & 0.51 & $0.49,0.54$ & $<0.05$ & 0.78 \\
\hline 1 & 0.31 & $0.30,0.33$ & $<0.05$ & & 0.47 & $0.45,0.49$ & $<0.05$ & \\
\hline 2 & 0.52 & $0.50,0.55$ & $<0.05$ & & 0.62 & $0.60,0.65$ & $<0.05$ & \\
\hline 3 & \multicolumn{3}{|c|}{ Reference } & \multicolumn{5}{|c|}{ Reference } \\
\hline 4 & 1.26 & $1.20,1.33$ & $<0.05$ & & $1 \cdot 17$ & $1 \cdot 11,1 \cdot 24$ & $<0.05$ & \\
\hline 5 & 1.60 & $1.50,1.72$ & $<0.05$ & & 1.38 & $1.28,1.49$ & $<0.05$ & \\
\hline 6 & 2.01 & $1 \cdot 75,2 \cdot 31$ & $<0.05$ & & 1.72 & $1 \cdot 48,2 \cdot 01$ & $<0.05$ & \\
\hline 7 & 4.69 & $3.73,5.89$ & $<0.05$ & & 3.05 & $2 \cdot 37,3.93$ & $<0.05$ & \\
\hline \multicolumn{9}{|c|}{ Disease severity score } \\
\hline 0 & \multicolumn{2}{|l|}{ Reference } & \multicolumn{6}{|c|}{ Reference } \\
\hline 1 & 0.96 & $0.93,0.99$ & $0 \cdot 19$ & 0.63 & 0.85 & $0.82,0.88$ & $<0.05$ & 0.78 \\
\hline 2 & 2.55 & $2 \cdot 46,2 \cdot 6$ & $<0.05$ & & 2.05 & $1.97,2 \cdot 13$ & $<0.05$ & \\
\hline 3 & $11 \cdot 1$ & $10.5,11 \cdot 7$ & $<0.05$ & & 1.74 & $1.52,2 \cdot 00$ & $<0.05$ & \\
\hline \multicolumn{9}{|c|}{ Nutrition status score } \\
\hline 0 & \multicolumn{3}{|l|}{ Reference } & \multicolumn{4}{|c|}{ Reference } & \\
\hline 1 & $2 \cdot 35$ & $2 \cdot 28,2 \cdot 42$ & $<0.05$ & 0.59 & 1.44 & $1.40,1.50$ & $<0.05$ & 0.78 \\
\hline 2 & $2 \cdot 16$ & $2 \cdot 04,2 \cdot 28$ & $<0.05$ & & 1.73 & $1 \cdot 62,1.84$ & $<0.05$ & \\
\hline 3 & $2 \cdot 00$ & $1 \cdot 90,2 \cdot 10$ & $<0.05$ & & 1.64 & $1.55,1.73$ & $<0.05$ & \\
\hline \multicolumn{9}{|l|}{ Albumin $(g / l)$} \\
\hline$>40$ & \multicolumn{3}{|l|}{ Reference } & 0.67 & \multicolumn{3}{|c|}{ Reference } & 0.78 \\
\hline $36-40$ & 1.90 & $1.83,1.97$ & $<0.05$ & & 1.61 & $1.55,1.68$ & $<0.05$ & \\
\hline $30-35$ & 3.41 & $3.28,3.55$ & & & $2 \cdot 21$ & $2 \cdot 11,2 \cdot 31$ & & \\
\hline$<30$ & 5.95 & $5 \cdot 72,6 \cdot 19$ & & & 2.98 & $2 \cdot 84,3 \cdot 12$ & & \\
\hline \multicolumn{9}{|l|}{ BMI $\left(\mathrm{kg} / \mathrm{m}^{2}\right)$} \\
\hline$<18.5$ & 1.38 & $1.30,1.46$ & $<0.05$ & 0.53 & 1.26 & $1 \cdot 18,1 \cdot 35$ & $<0.05$ & 0.78 \\
\hline $18 \cdot 5-23 \cdot 9$ & Reference & & & \multirow{2}{*}{\multicolumn{5}{|c|}{$\begin{array}{cc} & \text { Reference } \\
0.83 & 0.798,0.85\end{array}$}} \\
\hline $24-27 \cdot 9$ & 0.86 & $0.83,0.88$ & $<0.05$ & & & & & \\
\hline$>28$ & 0.89 & $0.86,0.93$ & $<0.05$ & & 0.84 & $0 \cdot 807,0 \cdot 88$ & $<0.05$ & \\
\hline \multicolumn{9}{|l|}{ Age (years) } \\
\hline$<69$ & \multicolumn{3}{|l|}{ Reference } & 0.539 & \multicolumn{3}{|c|}{ Reference } & 0.76 \\
\hline$\geq 70$ & 1.45 & $1.41,1.49$ & $<0.05$ & & 1.03 & $1.00,1.06$ & 0.30 & \\
\hline
\end{tabular}

patients were generally hospitalised longer and had lower survival rates ${ }^{(13,38)}$. Without effective intervention, AKI can develop irreversibly in patients with CKD and end-stage renal disease $^{(5)}$ and increase mortality. Although many guidelines recommend a good nutritional support in AKI patients, the increase of metabolic work for nutritional support may aggravate AKI progress ${ }^{(39)}$. Therefore, further investigation of the relationship of AKI and malnutrition is crucial.

Studies have demonstrated that malnutrition is related to poor prognosis in AKI, but no study has shown that malnutrition screening predicts the occurrence of AKI. Marina et al. enrolled 133 patients with a clinical diagnosis of AKI and identified that nutritional markers (a low energetic intake, higher C-reactive protein level, etc.) are significantly associated with a risk of death in AKI patients ${ }^{(40)}$. Fiaccadori et al. assessed 309 patients with AKI based on subjective global assessment (SGA); the results showed that $58 \%$ of patients had undernutrition and that severe undernutrition was associated with poor prognosis ${ }^{(41)}$. Our research confirms that the malnutrition risk (NRS-2002 scores $\geq 3$ ) is strongly associated with the development of AKI. The incidence of AKI was $29 \%$ in the NRS-2002 scores $\geq 3$ group, which was higher than $16 \%$ in the NRS-2002 <3 group. Furthermore, malnutrition, hypoproteinaemia, abnormal BMI levels, diabetes mellitus, chronic lung disease and other disease are recognised risk factors leading to $\mathrm{AKI}^{(42)}$. The individual factors are weak to predict the AKI, but as an entirety tool, NRS-2002 shows a robust ability to predict AKI. When the NRS-2002 score increased, the risk of AKI increased ( 0.67 AUC for the univariate and 0.78 for the multivariate model) and the association between the NRS2002 and AKI was constant across different sex, surgical status and history of diabetes mellitus and hypertension, which suggests that the association between the NRS-2002 and AKI has good generalisability in different clinical settings. Thus, clinicians should continuously test Scr or other biomarkers in undernutrition patients to determine whether renal function is impaired, avoid the use of nephrotoxic drugs and maintain blood perfusion of the kidneys.

The NRS-2002 has good generality and accuracy in evaluating the risk of long-term and short-term mortality, but whether NRS2002 scores $\geq 3$ or AKI independently affects long-term mortality is unknown. Almeida et $a l .{ }^{(43)}$ compared the subjective global assessment and NRS-2002 in their validation study conducted with 300 surgical patients. The results showed that the NRS2002 correctly identified $96 \%$ of the patients at nutritional risk compared with subjective global assessment. The sensitivity and specificity values for the NRS-2002 were $0.8(0.76-0.84)$ and $0.89(0.84-0.92)$, respectively, thus successfully identifying risk. Our results showed that both the NRS-2002 and AKI had 
(a)

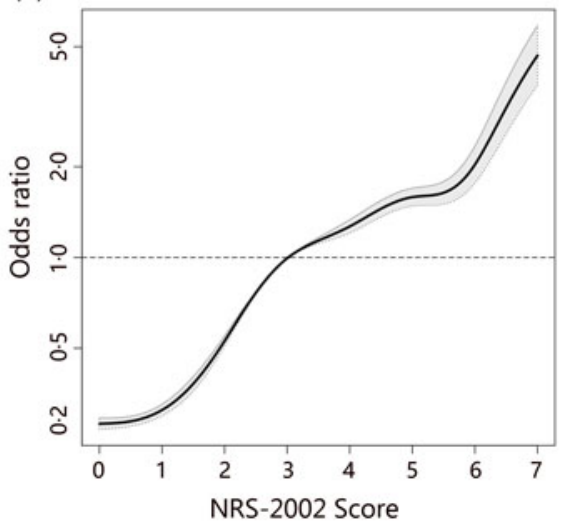

(d)

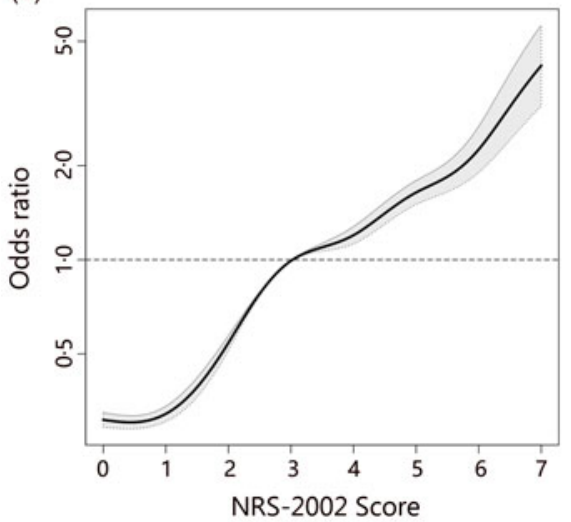

(g)

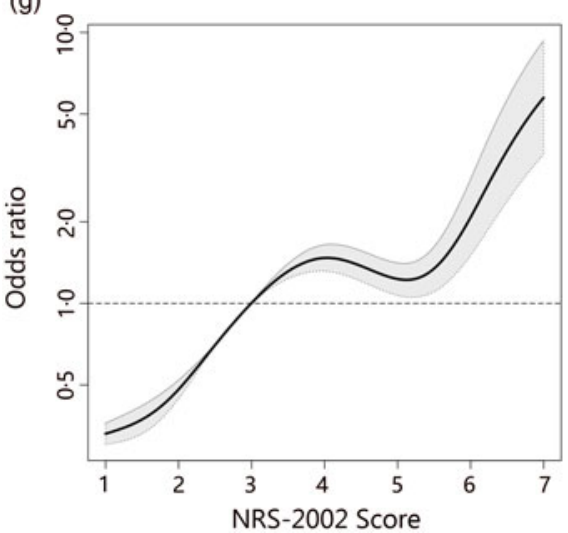

(b)

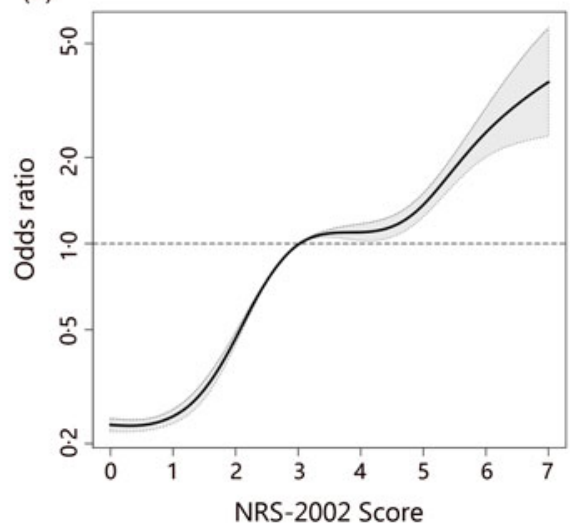

(e)

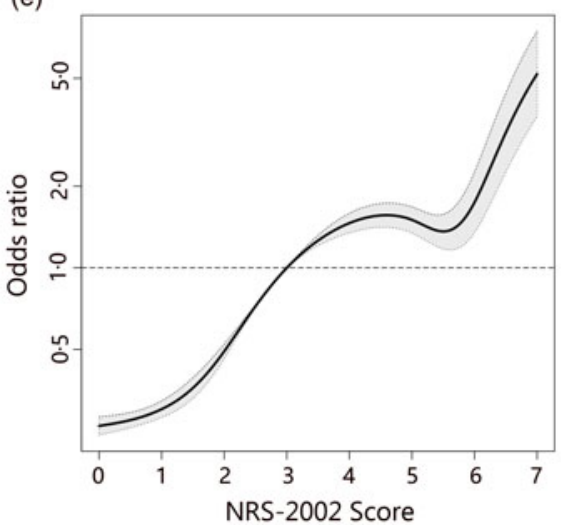

(h)

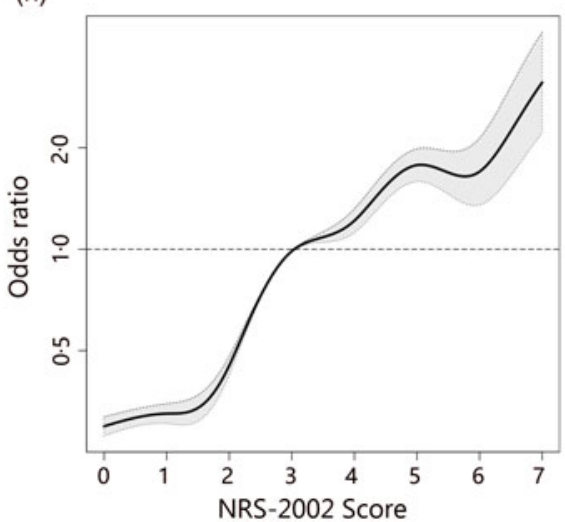

(c)

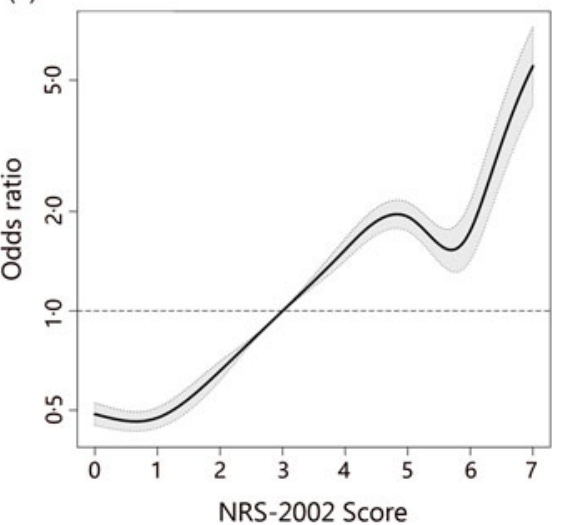

(f)

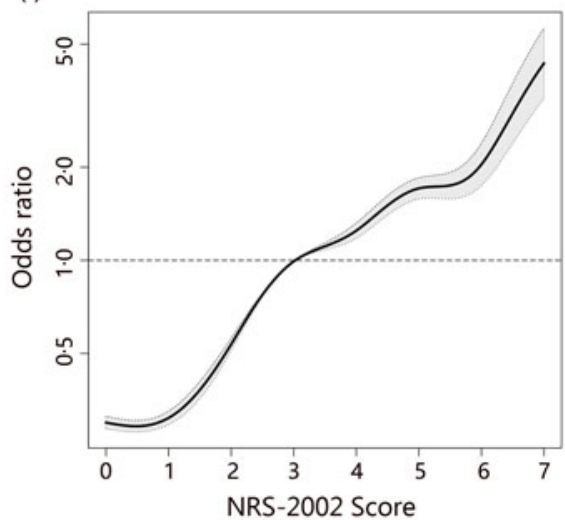

(i)

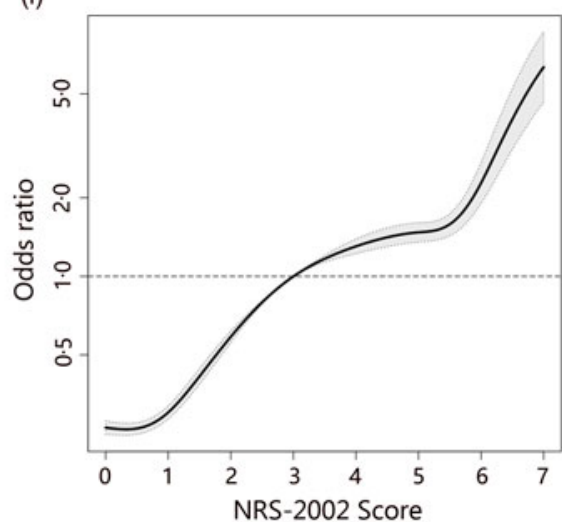

Fig. 2. Distributions and odds ratios of Nutritional Risk Screening 2002 (NRS-2002) for acute kidney injury (AKI) by using restricted cubic splines among 46549 inpatients. Logistic regression analysis with restricted cubic spline functions displayed a significant association of change in NRS-2002 with AKI in different clinical settings. (a) All patients; (b) non-surgery; (c) surgery; (d) non-hypertension; (e) hypertension; (f) non-diabetes; (g) diabetes; (h) male; (i) female.

a dose-response relationship between their severity and the adverse effect on the short- and long-term survival of the patients, which is consistent with previous studies ${ }^{(40,44,45)}$. AKI patients with NRS-2002 scores $\geq 3$ had the worst prognosis (HR 24.8, 23.3-26.3) than non-AKI patients with NRS-2002 scores $<3$ (HR 6.47, 6.06-6.90, $P<0.05$ ) or AKI patients with NRS-2002 scores $\geq 3(\mathrm{HR} 6 \cdot 74,6 \cdot 28-7 \cdot 24, P<0 \cdot 05)$, indicating that both malnutrition risk (NRS-2002 scores $\geq 3$ ) and AKI are adverse to prognosis of patients. Univariate Cox regression analyses showed that the NRS-2002 and AKI have a significant association with short- and long-term survival, while multivariate analyses showed that NRS-2002 scores $\geq 3$ were not the direct factor that increased mortality. Moreover, the NRS-2002 and AKI have synergy that better predict the long-term prognosis of patients with an AUC of 0.80, which is better than using either AKI (AUC 0.71) or NRS-2002 (AUC 0.79) alone. Our finding suggests that both NRS-2002 scores $\geq 3$ and AKI can worsen the short- and long-term prognosis of patients and the prognosis of the malnourished patient who develops AKI is far worse than that of the patient with normal nutritional status; thus, good nutritional support has been advocated in AKI.

We also found that ALB was an important nutritional variable associated with AKI (AUC 0.67). Low ALB is a known predictor of poor outcomes, including renal dysfunction and mortality ${ }^{(46)}$. 
Table 4. Adjusted and unadjusted Cox regression model of the association between Nutritional Risk Screening 2002/acute kidney infection (NRS-2002/AKI) and prognosis of patients

(Odds ratios and interquartile ranges (IQR))

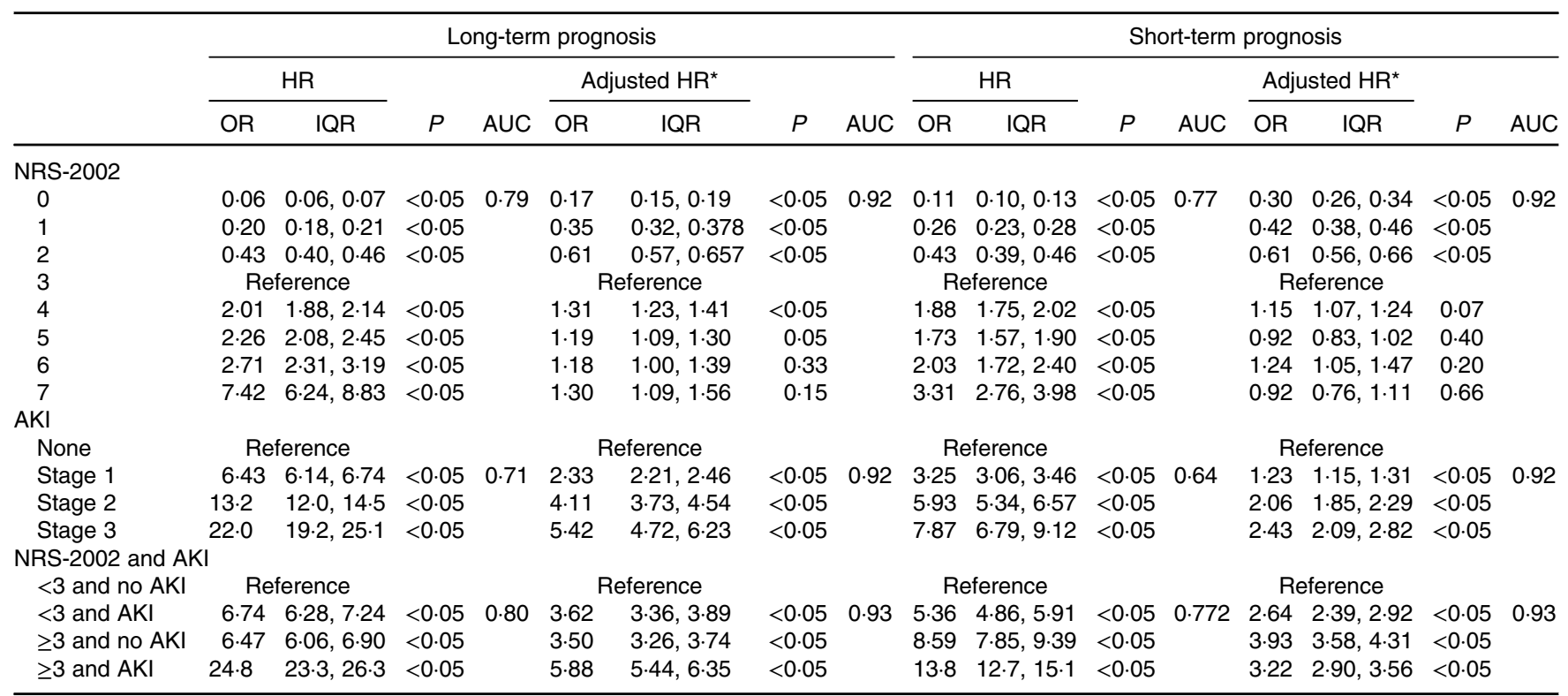

* The Cox regression model was adjusted by aspartate aminotransferase, uric acid, $\beta$-blockers, medical, sex, leucocytes, $\mathrm{Hb}$, proton pump inhibitors, antibiotic, oncology, nonsteroidal anti-inflammatory drugs, lactate dehydrogenase, $\mathrm{CHD}$, diabetes mellitus, and intensive care unit admission.

(a)

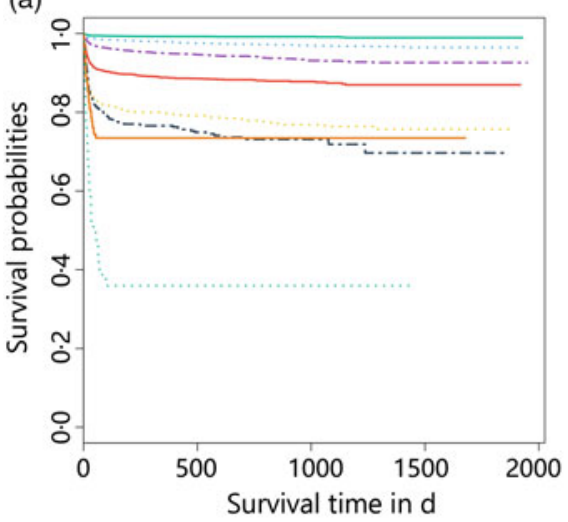

(d)

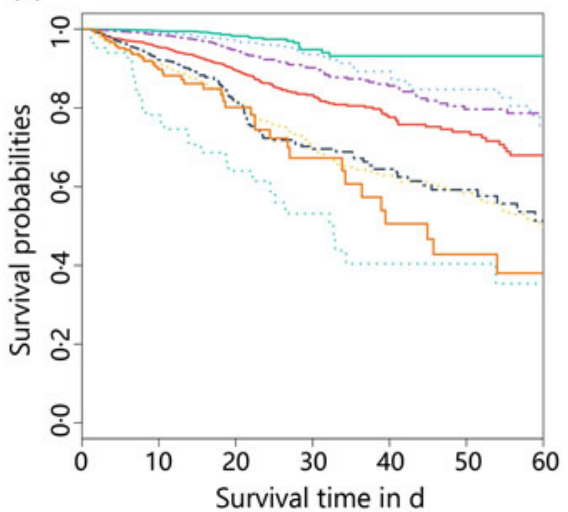

(b)

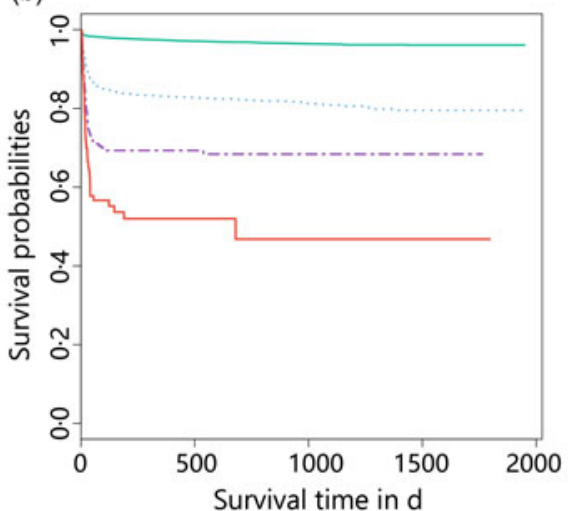

(e)

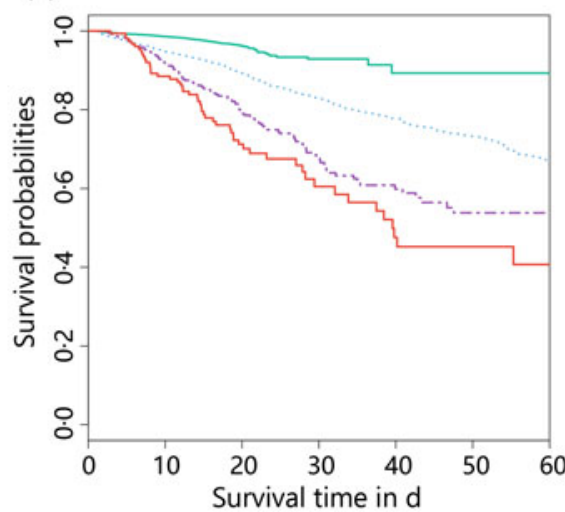

(c)

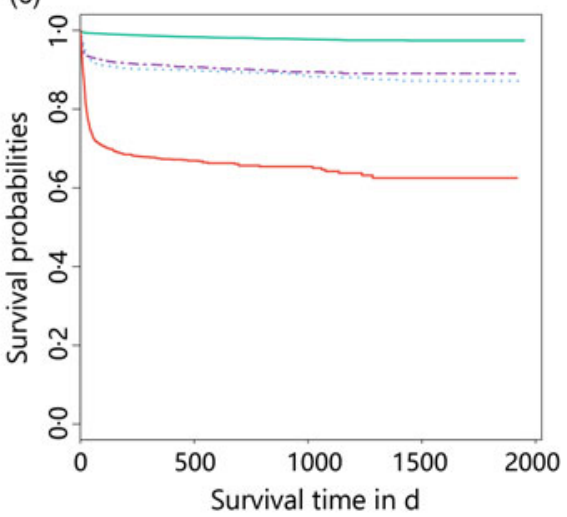

(f)

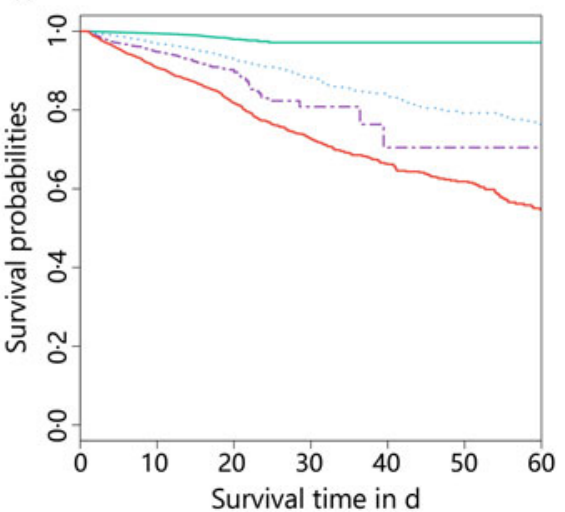

Fig. 3. Stratification of long- (a-c) and short- (d-f) term survival probabilities among patients in different Nutritional Risk Screening 2002 (NRS-2002) scores and acute

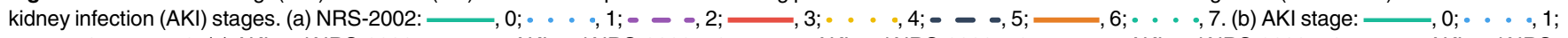

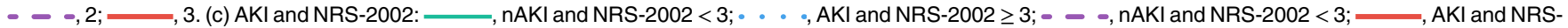

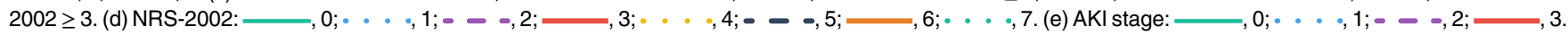
(f) AKI and NRS-2002: —, nAKI and NRS-2002<3; —, AKI and NRS-2002 $\geq 3$; —, nAKI and NRS-2002 <3; —, AKI and NRS-2002 $\geq 3$. 
Engelman et al. reported that hypoalbuminaemia was an independent risk factor for AKI after cardiac surgery ${ }^{(47)}$, which was consistent with our results. Other parts of the NRS-2002 (impaired nutritional status, severity of disease and age) slightly contributed to the risk of AKI but were not as significant as the total score of the NRS-2002. High BMI increases the risk of AKI; however, our results showed that BMI was not associated with AKI (AUC 0.526). A previous study showed that patients with AKI and a BMI of $30-35 \mathrm{~kg} / \mathrm{m}^{2}$ had a reduced risk of mortality during hospitalisation ${ }^{(48)}$. Furthermore, Qian et al. performed a meta-analysis including 2157 AKI cases and 49777 controls, showing that BMI was not absolutely associated with $\mathrm{AKI}^{(49)}$. Therefore, due to the difficulty in determining the dry weight of patients, using BMI as a parameter of nutritional monitoring in association with AKI is questionable.

The present study has several limitations. First, our data were obtained from five institutions. Thus, future studies are needed to validate our results in a broader setting. Second, the urine output was recorded by patients' family members, which was not accurate and excluded for further analysis in our study. Although we collected nearly all of the comprehensive indicators and used reasonable statistical methods to eliminate bias, variables with inaccurate and missing data, such as urine output, were not included in this analysis, which might have affected our results and the predictive efficacy of the model. Finally, Cox and logistic regression models are reliable classifications and prediction methods, but these approaches are not the best analytical methods considering the rapid development of machine learning such as Support Vector Machine and Deep-learning approach ${ }^{(50,51)}$, which may help attain better prediction results.

In conclusion, malnutrition is common among hospitalised patients and increases LOS and mortality. There is a strong association between NRS-2002 and AKI that the risk of AKI changed in parallel with the NRS-2002 score. Both AKI and NRS-2002 scores $\geq 3$ can worsen the prognosis that the undernourished patients who develop AKI yield far worse prognosis than patients with normal nutritional status. Additional studies are required to determine whether the NRS-2002 can be used in cases of AKI in other institutions or countries.

\section{Acknowledgements}

This work was supported by the National Natural Science Foundation of China (81170688, 81470973, 81770679 and 81800601). No funding bodies had any role in study design, data collection and analysis, decision to publish or preparation of the manuscript.

C. Li conceived of the presented idea and contributed to study design, data analysis and interpretation. L. X., C. G. and L. Z. verified the analytical methods and statistical results, and contributed to the final version of the manuscript. C. Luo, B. Z, X. Z., J. W., J. Z., J. H., D. L., H. L. and X. M. are independent members who collected the data within 5 years. L. C., Y. W. and H. Z. contributed to the interpretation of the results. Y.X. is the senior author of this manuscript in charge of overall direction and planning. All authors provided critical feedback and helped shape the research, analysis and manuscript.

The authors declare that there are no conflicts of interest.

\section{Supplementary material}

For supplementary material referred to in this article, please visit https://doi.org/10.1017/S000711451900271X

\section{References}

1. Coca SG, Yusuf B, Shlipak MG, et al. (2009) Long-term risk of mortality and other adverse outcomes after acute kidney injury: a systematic review and meta-analysis. Am J Kidney Dis $\mathbf{5 3}$, 961-973.

2. Rossaint J \& Zarbock A (2016) Acute kidney injury: definition, diagnosis and epidemiology. Minerva Urol Nefrol 68, 49-57.

3. Chawla LS, Bellomo R, Bihorac A, et al. (2017) Acute kidney disease and renal recovery: consensus report of the Acute Disease Quality Initiative (ADQI) 16 Workgroup. Nat Rev Nephrol 13, 241-257.

4. Silver SA \& Chertow GM (2017) The economic consequences of acute kidney injury. Nephron 137, 297-301.

5. Coca SG, Singanamala S \& Parikh CR (2012) Chronic kidney disease after acute kidney injury: a systematic review and meta-analysis. Kidney Int 81, 442-448.

6. Chawla LS, Eggers PW, Star RA, et al. (2014) Acute kidney injury and chronic kidney disease as interconnected syndromes. N Engl J Med 371, 58-66.

7. Curtis LJ, Bernier P, Jeejeebhoy K, et al. (2017) Costs of hospital malnutrition. Clin Nutr 36, 1391-1396.

8. Hartz LLK, Stroup BM, Bibelnieks TA, et al. (2019) ThedaCare Nutrition Risk Screen improves the identification of non-intensive care unit patients at risk for malnutrition compared with the Nutrition Risk Screen 2002. JPEN J Parenter Enteral Nutr 43, 70-80.

9. Kyle UG, Kossovsky MP, Karsegard VL, et al. (2006) Comparison of tools for nutritional assessment and screening at hospital admission: a population study. Clin Nutr 25 , 409-417.

10. Corkins MR, Guenter P, DiMaria-Ghalili RA, et al. (2014) Malnutrition diagnoses in hospitalized patients: United States, 2010. JPEN J Parenter Enteral Nutr 38, 186-195.

11. Makhija S \& Baker J (2008) The Subjective Global Assessment: a review of its use in clinical practice. Nutr Clin Pract 23, 405-409.

12. Malone A \& Hamilton C (2013) The Academy of Nutrition and Dietetics/the American Society for Parenteral and Enteral Nutrition consensus malnutrition characteristics: application in practice. Nutr Clin Pract 28, 639-650.

13. Borek P, Chmielewski M, Malgorzewicz S, et al. (2017) Analysis of outcomes of the NRS 2002 in patients hospitalized in nephrology wards. Nutrients $\mathbf{9}, 287$.

14. Xia YA, Healy A \& Kruger R (2016) Developing and validating a renal nutrition screening tool to effectively identify under nutrition risk among renal inpatients. J Ren Nutr 26, 299-307.

15. MacLaughlin HL, Twomey J, Saunt R, et al. (2018) The nutrition impact symptoms (NIS) score detects malnutrition risk in patients admitted to nephrology wards. J Hum Nutr Diet 31, 683-688.

16. Fiaccadori E, Maggiore U, Cabassi A, et al. (2013) Nutritional evaluation and management of AKI patients. J Ren Nutr $\mathbf{2 3}$, 255-258. 
17. Fiaccadori E, Cremaschi E \& Regolisti G (2011) Nutritional assessment and delivery in renal replacement therapy patients. Semin Dial 24, 169-175.

18. Mouser JF, Hak EB, Kuhl DA, et al. (1997) Recovery from ischemic acute renal failure is improved with enteral compared with parenteral nutrition. Crit Care Med 25, 1748-1754.

19. Pons M, Plante I, LeBrun M, et al. (2003) Protein-rich diet attenuates cyclosporin A-induced renal tubular damage in rats. J Ren Nutr 13, 84-92.

20. Roberts PR, Black KW \& Zaloga GP (1997) Enteral feeding improves outcome and protects against glycerol-induced acute renal failure in the rat. Am J Respir Crit Care Med 156, 1265-1269.

21. Abel RM, Beck CH, Jr., Abbott WM, et al. (1973) Improved survival from acute renal failure after treatment with intravenous essential L-amino acids and glucose. Results of a prospective, double-blind study. N Engl J Med 288, 695-699.

22. Doig GS, Simpson F, Bellomo R, et al. (2015) Intravenous amino acid therapy for kidney function in critically ill patients: a randomized controlled trial. Intensive Care Med $\mathbf{4 1}$, 1197-1208.

23. Fiaccadori E, Regolisti G \& Maggiore U (2013) Specialized nutritional support interventions in critically ill patients on renal replacement therapy. Curr Opin Clin Nutr Metab Care 16, 217-224.

24. Fiaccadori E, Sabatino A, Morabito S, et al. (2016) Hyper/ hypoglycemia and acute kidney injury in critically ill patients. Curr Opin Clin Nutr Metabolic Care 35, 317-321.

25. Kondrup J, Allison SP, Elia M, et al. (2003) ESPEN guidelines for nutrition screening 2002. Clin Nutr 22, 415-421.

26. Peng H, Chen BB, Tang LL, et al. (2018) Prognostic value of nutritional risk screening 2002 scale in nasopharyngeal carcinoma: a large-scale cohort study. Cancer Sci 109, 1909-1919.

27. Inoue T, Misu S, Tanaka T, et al. (2019) Acute phase nutritional screening tool associated with functional outcomes of hip fracture patients: a longitudinal study to compare MNA-SF, MUST, NRS-2002 and GNRI. Clin Nutr 38, 220-226.

28. Tan R, Long J, Fang S, et al. (2016) Nutritional Risk Screening in patients with chronic kidney disease. Asia Pac J Clin Nutr 25 , 249-256.

29. Kondrup JE, Allison SP, Elia M, et al. (2003) ESPEN guidelines for nutrition screening 2002. Clin Nutr 22, 415-421.

30. Levey AS, Stevens LA, Schmid CH, et al. (2009) A new equation to estimate glomerular filtration rate. Ann Intern Med 150, 604-612.

31. Jie B, Jiang ZM, Nolan MT, et al. (2012) Impact of preoperative nutritional support on clinical outcome in abdominal surgical patients at nutritional risk. Nutrition 28, 1022-1027.

32. Zhang H, Wang Y, Jiang ZM, et al. (2017) Impact of nutrition support on clinical outcome and cost-effectiveness analysis in patients at nutritional risk: a prospective cohort study with propensity score matching. Nutrition 37, 53-59.

33. Zhang Z (2016) Multiple imputation with multivariate imputation by chained equation (MICE) package. Ann Transl Med 4, 30 .

34. Ibrahim JG, Chu H \& Chen MH (2012) Missing data in clinical studies: issues and methods. J Clin Oncol 30, 3297-3303.
35. Schafer JL \& Graham JW (2002) Missing data: our view of the state of the art. Psychol Methods 7, 147-177.

36. Austin PC (2011) Optimal caliper widths for propensity-score matching when estimating differences in means and differences in proportions in observational studies. Pharm Stat 10, 150-161.

37. Stratton RJ, Hackston A, Longmore D, et al. (2004) Malnutrition in hospital outpatients and inpatients: prevalence, concurrent validity and ease of use of the 'Malnutrition Universal Screening Tool' ('MUST') for adults. Br J Nutr 92, 799-808.

38. Norman K, Pichard C, Lochs H, et al. (2008) Prognostic impact of disease-related malnutrition. Clin Nutr 27, 5-15.

39. Joannidis M, Druml W, Forni LG, et al. (2017) Prevention of acute kidney injury and protection of renal function in the intensive care unit: update 2017: expert opinion of the Working Group on Prevention, AKI section, European Society of Intensive Care Medicine. Intensive Care Med 43, 730-749.

40. Berbel MN, Goes CR, Balbi AL, et al. (2014) Nutritional parameters are associated with mortality in acute kidney injury. Clinics (Sao Paulo) 69, 476-482.

41. Fiaccadori E, Lombardi M, Leonardi S, et al. (1999) Prevalence and clinical outcome associated with preexisting malnutrition in acute renal failure: a prospective cohort study. $J$ Am Soc Nephrol 10, 581-593.

42. Hoste EAJ, Kellum JA, Selby NM, et al. (2018) Global epidemiology and outcomes of acute kidney injury. Nat Rev Nephrol 14, 607-625.

43. Almeida AI, Correia M, Camilo M, et al. (2012) Nutritional risk screening in surgery: valid, feasible, easy! Clin Nutr $\mathbf{3 1}$, 206-211.

44. Shiao CC, Huang YT, Lai TS, et al. (2017) Perioperative body weight change is associated with in-hospital mortality in cardiac surgical patients with postoperative acute kidney injury. PLOS ONE 12, e0187280.

45. Hobson CE, Yavas S, Segal MS, et al. (2009) Acute kidney injury is associated with increased long-term mortality after cardiothoracic surgery. Circulation 119, 2444-2453.

46. Engelman DT, Adams DH, Byrne JG, et al. (1999) Impact of body mass index and albumin on morbidity and mortality after cardiac surgery. J Thorac Cardiovasc Surg 118, 866-873.

47. Lee EH, Baek SH, Chin JH, et al. (2012) Preoperative hypoalbuminemia is a major risk factor for acute kidney injury following off-pump coronary artery bypass surgery. Intensive Care Med 38, $1478-1486$.

48. Druml W, Metnitz B, Schaden E, et al. (2010) Impact of body mass on incidence and prognosis of acute kidney injury requiring renal replacement therapy. Intensive Care Med 36, 1221-1228.

49. Yi Q, Li K, Jian Z, et al. (2016) Risk factors for acute kidney injury after cardiovascular surgery: evidence from 2,157 cases and 49,777 controls - a meta-analysis. Cardiorenal Med 6, 237-250.

50. Esteva A, Kuprel B, Novoa RA, et al. (2017) Corrigendum: Dermatologist-level classification of skin cancer with deep neural networks. Nature 546, 686.

51. Esteva A, Kuprel B, Novoa RA, et al. (2017) Dermatologist-level classification of skin cancer with deep neural networks. Nature 542, 115-118. 